\title{
Controversy over renin-angiotensin-aldosterone system (RAAS) inhibitors treatment in nephrology and cardiovascular diseases
}

\author{
Rafał Donderski $\odot$, Rafał Bednarski $\odot$, Jacek Manitius $\odot$ \\ Department of Nephrology, Hypertension and Internal Medicine, Ludwik Rydygier Collegium Medicum in Bydgoszcz, \\ Nicolaus Copernicus University in Toruń, Poland
}

\begin{abstract}
Drugs that act by inhibiting the renin-angiotensin-aldosterone system (RAAS), such as angiotensin-converting enzyme inhibitors (ACE-Is) and angiotensin II receptor type 1 (AT1) blockers (ARBs), have been recognized as a basic canon of nephroprotection for years. They are commonly used in monotherapy for glomerulonephritis with proteinuria. At present, they are rarely used in combination therapy in a form of dual blockade of RAAS due to concern about possible side effects. On the other hand, both ACE-Is and ARBs are also wrongly referred to as nephrotoxic drugs. The significance of therapy with these drugs is seen in evoking acute kidney injury (AKI) or acceleration of CKD. The aim of this article was to clarify the opinion on the relationship between ACE-Is or ARBs treatment and AKI occurrence, and to attempt to reassess the role of dual RAAS blockade in the treatment of kidney diseases. The principles of heart failure (HF) therapy with ACE-Is or ARBs and current data on the importance of RAAS dual blockade in hypertension are also discussed.
\end{abstract}

Key words: acute kidney injury; chronic kidney disease; renin-angiotensin-aldosterone system; dual RAAS blockade; halting the progression of kidney disease

Arterial Hypertens. 2020, vol. 24, no. 2, pages: 45-55

DOI: $10.5603 /$ AH.a2020.0007

\section{Angiotensin-converting enzyme inhibitors (ACE-Is) and angiotensin II receptor type 1 (AT1) blockers (ARBs) and their relationship with acute kidney injury (AKI)}

Inhibition of the renin-angiotensin-aldosterone system (RAAS) using angiotensin-converting enzyme inhibitors (ACE-Is) or angiotensin II receptor type 1 (AT1) blockers (ARBs) is the basic canon of nephroprotection that has been used for years in proteinuric kidney disease treatment. These drugs inhibit the progression of chronic nephropathy to the end stage renal failure and increase the time to renal replacement therapy initiation. Both ACE-Is and ARBs exhibit multidirectional properties and can improve prognosis in patients with coronary artery disease, especially after myocardial infarction, hypertensive patients or individuals after stroke $[1,2]$. Because of cardioprotective, angioprotective and nephroprotective potential of these drugs, they are often prescribed also in patients with heart failure (HF), with both preserved and reduced ejection fraction $(\mathrm{EF})$, and coexisting chronic kidney disease (CKD) [3].

Address for correspondence: Dr hab. med. Rafał Donderski,

Department of Nephrology, Hypertension and Internal Medicine, Ludwik Rydygier Collegium Medicum in Bydgoszcz,

Nicolaus Copernicus University in Toruń, Dr A. Jurasz University Hospital No. 1, ul. Skłodowskiej-Curie 9, 85-094 Bydgoszcz, Poland, tel: (+48) 05258540 30, fax (+48) 05258540 30; e-mail: rafdon@o2.pl 
However, there is also a common conviction that ACE-Is or ARBs treatment may cause acute kidney injury (AKI) or deterioration of pre-existing CKD in certain clinical situations. In elderly patients with concomitant $\mathrm{CKD}$, after an $\mathrm{AKI}$ episode, in population with atherosclerotic cardiovascular disease, initiation of ACE-Is or ARBs therapy may cause deterioration of renal function expressed by increased serum creatinine level. This is clinical scenario has been known for years and has been associated with the effect of these drugs on glomerular haemodynamics and reduction of intra-glomerular capillary pressure. The occurrence of AKI shortly after the commencement of ACE-Is therapy is a well-known diagnostic marker for renal artery stenosis. In elderly patients, ACE-Is or ARBs treatment should be administered with caution, starting with low doses; and preferably renal artery stenosis should be excluded before initiating the treatment in available imaging studies (renal arteries Doppler ultrasound or angio-CT).

In spite of primary cause of AKI, discontinuation of ACE-Is or ARBs treatment to stop further possible reduction of glomerular filtration rate, seems to be common clinical practice. This approach can be justified in patients with hypotension, hypovolaemia, in severe general condition, with onset of septic or cardiogenic shock. Such individuals require fluid resuscitation, starting treatment with catecholamines or broad-spectrum antibiotics, discontinuation of antihypertensive drugs, intubation and respiratotherapy or initiation of renal replacement therapy. On the other hand, in some patients with AKI, but in a good general condition, without hypotension, ACE-Is or ARBs treatment should be continued. It seems especially important, because increased RAAS activity, enhanced inflammation and oxidative stress are commonly observed in AKI. Pleiotropic properties of these drugs and inhibition of inflammation and oxidative stress may be considered to be a form of prevention of further renal fibrosis after AKI episode.

If a decision on discontinuation of ACE-Is or $A R B s$ in a patient previously chronically treated with one of these drugs has been made, it is important to establish, when the treatment should be reapplied as well as by whom and where the therapy should be conducted (i.e. restarting treatment by a nephrologist in the hospital ward or outpatient clinic, resumption of treatment by a family doctor). Taking into account the long-term benefits of ACE-Is or ARBs administration, it seems crucial to reapply this treatment as soon as possible. Gayat et al. in analysis of patients with AKI (who were hospitalized at Intensive Care Unit (ICU)) and were restarted ACE-Is or ARBs treatment on the day of ICU discharge, reported a statistically significant reduction in 1-year mortality in the ACE-Is or ARBs group compared with patients who did not receive this medication [4]. The authors suggest that ACE-Is or ARBs treatment should be considered for all patients discharged from ICUs as a preventive strategy against cardiovascular complications. It should be emphasized that ACE-Is or ARBs reintroduction should also be started with small doses of the drug, which can be gradually increased. Monitoring of serum creatinine level (expressed as eGFR) and serum potassium level is mandatory. An increase in creatinine $\geq 30 \%$ of baseline or a decrease in eGFR $\geq 25 \%$ is not an indication for discontinuation. An increase in serum creatinine concentration in the range of $30-50 \%$ can only indicate a need of $50 \%$ reduction of the daily dose of the drug. An increase in serum creatinine concentration $>50 \%$ requires discontinuation of the drug. In fact, the increase in serum creatinine level after switching on the drug indicates that the drug has nephroprotective effects, which should be remembered [3].

The exaggerated concern of further deterioration of renal function in patients with decompensation of congestive heart failure (CHF) may cause in some cases ACE-Is or ARBs discontinuation and, what is worse, sometimes these agents are not considered in further treatment, i.e. in stable period of CHF. In these cases, it seems better to continue therapy because the improvement of heart function as a result of ACE-Is or ARBs administration can undoubtedly contribute to the improvement of kidney function. This issue will be discussed further in this article.

There is also erroneous conviction that ACE-Is or ARBs are "nephrotoxic" or "potentially nephrotoxic" drugs. Such term can be found, for example, in the American Recommendations for the Diagnosis and Treatment of AKI. The KDIGO 2012 Guidelines (Guideline 4.4.3) recommend clinicians to carefully analyse the current pharmacotherapy in patients at high risk of AKI (e.g. CKD patients at stages G3a-G5) and consider temporary discontinuation of ACE-Is or ARBs in some clinical situations, e.g. before exposition to contrast agents or exacerbation of any concomitant chronic diseases such as already mentioned CHF. In the 'sick day approach' (sick day rules) recommended by the British National Institute for Health and Care Excellence (UK NICE Guidelines) and popularized among family doctors, it is recommended to discontinue ACE-Is, ARBs, metformin, diuretics, NSAIDs treatment in any acute situation (intercurrent illness) such as vomiting, diarrhoea, surgery, or before any medical procedure. However, an important question arises whether a pa- 
tient will have the ACE-Is or ARBs reapplied after the acute illness is resolved, especially in case of an elderly individuals with a high cardiovascular risk. Frequently, this treatment is not restored again, because of already mention fear of worsening glomerular filtration or hyperkalaemia. It is worth dispelling the myth of alleged nephrotoxicity of ACE-Is or ARBs as this still commonly used term should be replaced by the term: eGFR changes dependent on haemodynamic changes related with the influence of ACE-Is or ARBs. These haemodynamic changes, which are commonly known, appear due to the vasodilatation effect of these drugs on glomerular arterioles. This causes a decrease in intra-glomerular pressure and deterioration of glomerular filtration, especially in the elderly patients, with CKD and long-lasting hypertension, as well as impaired mechanisms of intrarenal blood flow. Therefore, ACE-Is or ARBs cannot be referred to as "nephrotoxic" drugs because the effects of their haemodynamic activity leading to a decrease in eGFR are fundamentally different from, for example direct renal tubules damage (direct nephrotoxic effect) caused by e.g. aminoglycosides antibiotics, radiocontrast agents, antiretroviral drugs or some chemotherapeutics drugs (cisplatin analogues) [5-7].

It also seems necessary to re-establish the current standards of preventing of contrast induced nephropathy (CIN) in terms of continuation or discontinuation of agents that inhibit the RAAS. CIN is an example of iatrogenic AKI that develops within 48-72 hours after parenteral contrast media administration. It is more common in elderly patients with pre-existing CKD, cardiovascular diseases, diabetes mellitus, females and on non-steroidal anti-inflammatory drugs (NSAIDs), diuretics or metformin treatment. ACE-Is and ARBs are included on the list of drugs considered to have adverse effects on CIN course. Therefore, in protocols on prevention of CIN occurrence or in the Guidelines of Radiological Societies, it is recommended to discontinue ACE-Is and ARBs $48 \mathrm{~h}$ before exposure to the contrast agent. It seems that currently there is no convincing evidence that discontinuation of ACE-Is or ARBs prior to exposure to the contrast agent may contribute to reducing the incidence of CIN. Therefore, this procedure raises more and more controversy [8].

Another important clinical problem is the management of ACE-Is/ARBs in patients undergoing surgery (treatment discontinuation vs treatment continuation). In the analysis of 1594 cases of patients undergoing cardiac surgery, Coca et al. compared groups of patients in whom ACE-I (or previously used ARB) was discontinued on the day of surgery and a group of patients in whom such treatment was continued. The researchers observed in the postoperative period a statistically significant increase in the serum creatinine concentration compared to the baseline before the surgery, especially in the group of patients who continued ACE-Is or ARBs treatment. At the same time, there were no differences in the levels of markers of structural kidney damage (NGAL, KIM-1, IL-18) between the group of patients who continued the treatment and the group where administration of ACE-I or ARB was stopped. The results of these studies indicate that the reduction of eGFR in patients treated with ACE-Is or $A R B s$ is functional, reversible and does not result from actually structural kidney damage. Moreover, in this group of patients, the appearance of AKI in the postoperative period may be related with hypotension resulting from the effect of an aesthetics, antibiotic therapy or NSAIDs, used after surgery for pain relief [9]. In addition, Whiting et al. in a metaanalysis of 6 clinical trials (3 randomized trials and 3 prospective cohort studies) involving 1663 patients did not find a justification for a need to discontinue ACE-Is or ARBs treatment as an AKI prevention strategy in patients undergoing coronary angiography or cardiac surgery [10]. Similar conclusions were presented by the authors of a meta-analysis published in 2018, involving 9 clinical trials in patients $(\mathrm{n}=6022)$ previously treated with ACE-I or ARB who underwent noncardiac surgery. The authors of the meta-analysis compared the strategy based on discontinuation of ACE-I or ARB therapy on the day of surgery vs. maintenance of ACE-I or ARB treatment on the day of surgery and during postoperative period. The authors noted a higher incidence of hypotension episodes in the perioperative period in patients who continued ACE-I or ARB treatment. However, no relationship was found between continuation of ACE-I or ARB treatment and the impact of this approach on the incidence of cardiovascular events and deaths due to cardiovascular causes [11]. As the authors conclude, there is a need for randomized trials to determine the relationship between the use of ACE-Is or ARBs in the pre- and postoperative period and the impact of such treatment on cardiovascular mortality.

In summary, it can be stated that:

- both ACE-Is and ARBs are not kidney damaging drugs. The mechanisms of their action and haemodynamic effects in renal microcirculation (decrease in intra-glomerular pressure) cause a reversible decrease in glomerular filtration;

- a distinction should be made between functional (depending on changes in intrarenal blood flow) and structural type of acute kidney injury (AKI) 
using available biomarkers for early kidney damage, which should be used more widely in clinical practice;

- long-term use of ACE-Is as well as ARBs after an AKI incident seems to be beneficial and contributes to a long-term reduction of cardiovascular mortality. It is also beneficial in terms of inhibiting kidney fibrosis after the AKI episode and constitutes CKD prevention.

\section{Dual RAAS blockade in kidney diseases. Is there still a place for it in contemporary nephrology?}

Since the introduction of ACE-Is in the 1980s and the emergence of ARBs in the mid-1990s, these drugs have become the basis for the treatment of patients with cardiovascular and kidney diseases. Both ACE-Is and ARBs have an established position as drugs that are a key element of pharmacological nephroprotection, especially in groups of patients with proteinuric primary glomerulonephritis.

The beneficial effect of both ACE-Is and ARBs is associated with not only systemic blood pressure decrease, but also intra-glomerular pressure decrease, which, as already mentioned, is associated with a reduction in proteinuria and halting of glomerular filtration rate decline. These two mechanisms play a crucial role in preventing progression of chronic kidney disease (CKD) to irreversible end-stage phase [12]. Simultaneously with haemodynamic effects, both ACE-Is and ARBs exert a number of beneficial non-haemodynamic actions by limiting inflammation, inhibiting renal interstitial fibrosis, inhibiting platelet aggregation, and apoptosis. Moreover, they improve endothelial function. ACE-Is as well as ARBs used alone reduce cardiovascular morbidity and mortality in the general population and in patients with kidney diseases. The beneficial effects and definite advantages over other drugs resulting from blocking the RAAS as mentioned earlier, caused that ACE-Is and sartans became the basis standard of nephroprotection. Ground-breaking clinical trials such as the REIN study with ramipril (Ramipril efficacy in nephropathy), the AASK study (African American Study of Kidney Disease and Hypertension), or the BENEDICT study (Bergamo nephrologic diabetes complication trial) conducted in patients with primary glomerulopathies with proteinuria and diabetic nephropathy, where ACE-Is therapy was used alone, showed a clear beneficial effect of ACE-Is on primary 'nephrological' endpoints such as delayed doubling of creatinine, delayed progression to the end-stage renal failure, or reduction of cardiovascular death [13-15]. Equally favourable results were obtained in the studies in which ARBs were used (IDNT, RENAAL, DETAIL) [16-18].

On the other hand, it is known that using the recommended therapeutic doses of ACE-Is or ARBs in monotherapy, it is often impossible to achieve full blockade of the RAAS. Inhibition of RAAS activity and reduction of the synthesis of e.g. aldosterone are usually observed during the first months of ACE-Is or ARBs monotherapy. As a result of the aldosterone escape phenomenon (aldosterone breakthrough) long-term administration of ACE-Is or ARBs does not further reduce aldosterone synthesis $[8,9]$. It seems that this problem affects about $30-50 \%$ of patients treated with ACE-Is or ARBs. It is presumed that one of its mechanisms is hyperkalaemia (associated with the use of these drugs) that stimulates the release of aldosterone or the lack of sufficient inhibition of AT II synthesis during ACE-Is therapy [19-21].

It is worth remembering that inhibition of angiotensin converting enzyme (ACE) causes an increase in the synthesis of angiotensin I (AT I), which is converted into angiotensin II (AT II) through a pathway independent of the converting enzyme (chymase). Comprehensive RAAS control requires a combination of ACE-I with ARB or the use of other drugs, e.g. mineralocorticoid receptor antagonists (MRAs) or direct renin inhibitors (DRIs). The association of ACE-I with ARB became the basis of the concept of so-called dual RAAS blockade. Rarely, although such treatment regimens were used in kidney diseases, DRI or MRA (triple RAAS blockade) were added to the combination of ACE-I with ARB. A method that was also used to inhibit the increased activity of the RAAS, although it did not find wider application, was the use of high doses of ARBs in monotherapy. High doses (ultra-high doses) were 2-3 times higher than those recommended in the treatment of hypertension and they allowed successful reduction of proteinuria $[22,23]$. Studies in animal models have shown that the use of high doses of ARBs caused reversibility of glomerulosclerosis [24].

Dual blockade has its rational premises. It uses 2 drugs with different mechanisms of action to achieve the intended effect - reduction of proteinuria. It allows the use of slightly smaller doses of each drug individually when starting the therapy. On the other hand, it allows flexible increase of the dose of each drug separately. In turn, dual blockade in which an aldosterone antagonist is attached to ACE-I or ARB prevents aldosterone escape and limits its adverse effects. Considering the harmful effects of aldo- 
sterone on the cardiovascular system (intensification of cardiac and vascular fibrosis) and the fact that it is also a recognized factor of the progression of $\mathrm{CKD}$, this seems completely justified, but it is not widely used due to the risk of hyperkalaemia.

It is worth remembering that the excess of aldosterone causes a weakening of the beneficial effect of ACE-Is on left ventricular (LV) hypertrophy (refractory LV hypertrophy) and an impairment of the nephrotective effect of ACE-I or ARB monotherapy [25]. The benefits of using the dual blockade have been found in a number of experimental studies. In animal models of diabetic and non-diabetic kidney disease, co-administration of ACE-I with sartan was shown to be more effective in reducing proteinuria and inhibiting structural changes in the kidney, more efficiently than when using these drugs alone. Although clinical trials showed a greater reduction in proteinuria in dual therapy, there are few data on the inhibition of CKD progression to end-stage phase due to dual blockade [26, 27].

The first clinical study showing the beneficial effects of dual therapy based on the combined use of ACE-I and ARB was the study with the acronym CALM (The candesartan and lisinopril microalbuminuria study) conducted in patients with type $2 \mathrm{di}$ abetes [28]. Two hundred patients with type 2 diabetes, hypertension and albuminuria, who were treated with candesartan $16 \mathrm{mg}$ daily, lisinopril $20 \mathrm{mg}$ daily or a combination of both, participated in this 12 -week multicentre trial. After 12 weeks of treatment, a beneficial effect of dual therapy i.e. statistically significant reduction in blood pressure and proteinuria (urinary albumin/creatinine ratio) compared to treatment with lisinopril or candesartan alone was found. The results of this study and the subsequent CALM2 study formed the basis for widespread use and recommendation of RAAS dual blockade in nephrology. This type of management was common for many years in patients with glomerulonephritis with proteinuria. The results of three studies, ONTARGET, ALTITUDE and VA-NEPHRON-D, reduced the enthusiasm with which the dual blockade was used in kidney diseases.

In the ONTARGET (Ongoing Telmisartan Alone and in combination with ramipril global endpoint trial) study, which involved 25620 patients with cardiovascular disease, the effects of ramipril and telmisartan monotherapy were compared to dual blockade with both agents. In the dual treatment group, there was a greater reduction in blood pressure and a greater decrease in albuminuria. On the other hand, an increased incidence of hypotension and AKI (including the need for renal replacement therapy) as well as an increased incidence of life-threatening hyperkalaemia were found. At the same time, no differences in cardiovascular mortality between the dual blockade and monotherapy with ramipril or telmisartan were demonstrated [29].

However, the ALTITUDE (The Aliskiren Trial in Type 2 Diabetes Using Cardiorenal Endpoints) study, comparing the effectiveness of the combination of aliskiren with ACE-I or ARB versus ACE-I or $A R B$ monotherapy in patients with diabetic kidney disease and proteinuria was prematurely terminated due to occurrence of kidney function deterioration, hypotension and hyperkalaemia in 69\% in patients treated with aliskiren (dual blockade arm) [30].

In the VA-NEPHRON-D (Veterans Affairs Nephropathy in Diabetes) study, in a group of patients with diabetic nephropathy with proteinuria in the course of type 2 diabetes in which concomitant treatment: losartan with lisinopril was used, 34\% reduction in the risk of glomerular filtration decline, reaching end-stage CKD or death compared to the losartan monotherapy was found. However, no statistical significance was achieved $(p=0.07)$, perhaps due to the fact that this study was prematurely terminated because of more frequent AKI and hyperkalaemia incidents in the dual blockade group [31].

Negative results of these clinical trials resulted in a decrease in interest in the treatment based on RAAS dual blockade. The last two mentioned studies were prematurely terminated due to numerous side effects in the dual therapy group. The shortening of the study duration provided for in the protocol does not allow determining the role of dual therapy in inhibiting CKD progression. These studies also showed no difference in cardiovascular mortality between the RAAS dual blockade group and the monotherapy group.

The results of these clinical trials resulted in a reduction and eventually discontinuation of the use of dual blockade, which was also included in the warnings issued in the United States by the Food and Drug Administration (FDA, 2012) and in Europe by the European Medicines Agency (EMA, 2014).

The key to understanding the adverse results of the aforementioned clinical trials, i.e. increasing the frequency of side effects in the dual blockade group, is selection of the study population. Elderly patients with high cardiovascular risk, including heart failure, patients susceptible to hypotension, on concomitant diuretic therapy, participated in these studies, in particular in the Ontarget study. In the Ontarget trial, $24 \%$ of patients enrolled in the study had CKD. The selection of the study population may have contrib- 
Table 1. Clinical situations in which it is reasonable to consider the dual blockade of RAAS, in case of ineffectiveness of monotherapy in kidney diseases patients

\begin{tabular}{|l|}
\hline 1. Younger patients (no reduction of proteinuria on monotherapy with \\
RAAS inhibitors and failure to achieve target blood pressure values) \\
\hline 2. Patients with non-diabetic glomerulopathies \\
\hline 3. Concomitant hypertension \\
\hline 4. Diagnosed albuminuria/proteinuria \\
\hline 5. Preserved renal excretory function \\
\hline 6. Excluded renal vascular disease \\
\hline 7. High level of patient's adherence to a low-potassium diet \\
\hline
\end{tabular}

uted to the adverse results, i.e. the mentioned adverse effects in the RAAS dual blockade arm. The ONTARGET post hoc analysis showed a reduction in proteinuria in the dual blockade arm, but only 13\% of participants had albuminuria and $4 \%$ - proteinuria. This study was assumed to be a study in a group of patients with high cardiovascular risk, and it was not a strict sense study evaluating already mentioned nephrological hard endpoints. Dual blockade of the RAAS (despite the aforementioned restrictions) should still be considered in the treatment of kidney diseases and it is worth to return to it again. However, this is not a treatment that can be offered to every patient with CKD. It is reasonable to consider RAAS dual blockade treatment only in a group of carefully selected patients [32]. Clinical situations in which the dual blockade of RAAS can be offered are shown in Table 1.

Finally, it is worth mentioning that in $2016 \mathrm{~Pa}$ levsky et al. published a re-analysis of the mentioned VA-NEPHRON-D study. The authors again demonstrated an increased incidence of AKI in patients receiving combination therapy (ACE-I + ARB) compared to monotherapy, but at the same time the dual treatment group showed faster renal function recovery, lower cardiovascular mortality and a lower risk of progression of kidney disease [33].

To sum up, RAAS dual blockade undoubtedly allows further reduction of proteinuria and has an advantage in this regard over monotherapy. On the other hand, there is no clear data from available clinical trials showing its effect on "renal" hard endpoints such as doubling of creatinine, time to starting dialysis or cardiovascular mortality. However, taking into account the role of proteinuria in the intensification of renal fibrosis processes and undoubtedly in the progression of $\mathrm{CKD}$, all actions aimed at reducing proteinuria including the use of a dual blockade in the selected groups of patients should be considered.

\section{Treatment with RAAS-blocking drugs in patients with heart failure}

Heart failure (HF) is a set of symptoms that may be accompanied by abnormalities in physical examination due to changes in the structure and/or function of a heart. The prevalence of HF, depending on the adopted definition, is estimated at $1-2 \%$ of the adult population in developed countries. In the age group over 70 it affects over $10 \%$ of the population [34]. Heart failure is a significant medical, social and economic problem. Despite modern therapy, the prognosis of patients with HF remains poor.

The goals of treatment in patients with HF include improving their clinical condition, exercise capacity and quality of life, preventing hospitalizations and reducing total mortality rate. ACE-Is were shown to reduce mortality and morbidity in patients with HF especially with reduced left ventricular ejection fraction ( $\mathrm{HFrEF}$ ) and are recommended for all symptomatic patients who have no contraindications and intolerance. In addition, ACE-Is are recommended in people with asymptomatic left ventricular systolic dysfunction to reduce the risk of developing HF, hospitalization due to HF and death [34].

Undoubtedly, the CONSENSUS study turned out to be a breakthrough for drugs of this group. The program involved 253 patients with congestive heart failure in NYHA class IV, randomized to enalapril or placebo. Six months later, the study was discontinued after $44 \%$ mortality in the placebo group and $26 \%$ in the enalapril group [35]. If ACE-Is are not tolerated or are contraindicated, ARBs should be used in these patients [34]. There are some restrictions regarding the use of $\mathrm{ACE}-\mathrm{Is}$ and $\mathrm{ARB}$ s in patients with $\mathrm{HF}$ accompanied by AKI or CKD. Patients with eGFR $<30 \mathrm{~mL} / \mathrm{min} / 1.73 \mathrm{~m}^{2}$ were excluded from clinical trials; therefore, in this group of patients there is no therapy with proven effectiveness based on scientific evidence [34].

$\mathrm{HF}$ is a risk factor for the development of CKD and these two conditions often coexist. Approximately $1 / 3$ of patients with heart failure have mild to moderate CKD and about $1 / 4$ of them have worsening renal function (WRF) during hospitalization due to heart failure [36]. While CKD is an independent risk factor for adverse prognosis in patients with HF, the importance of transient WRF in patients hospitalized for heart failure has not been fully established. In a meta-analysis by Damman et al. [37] involving over one million patients 


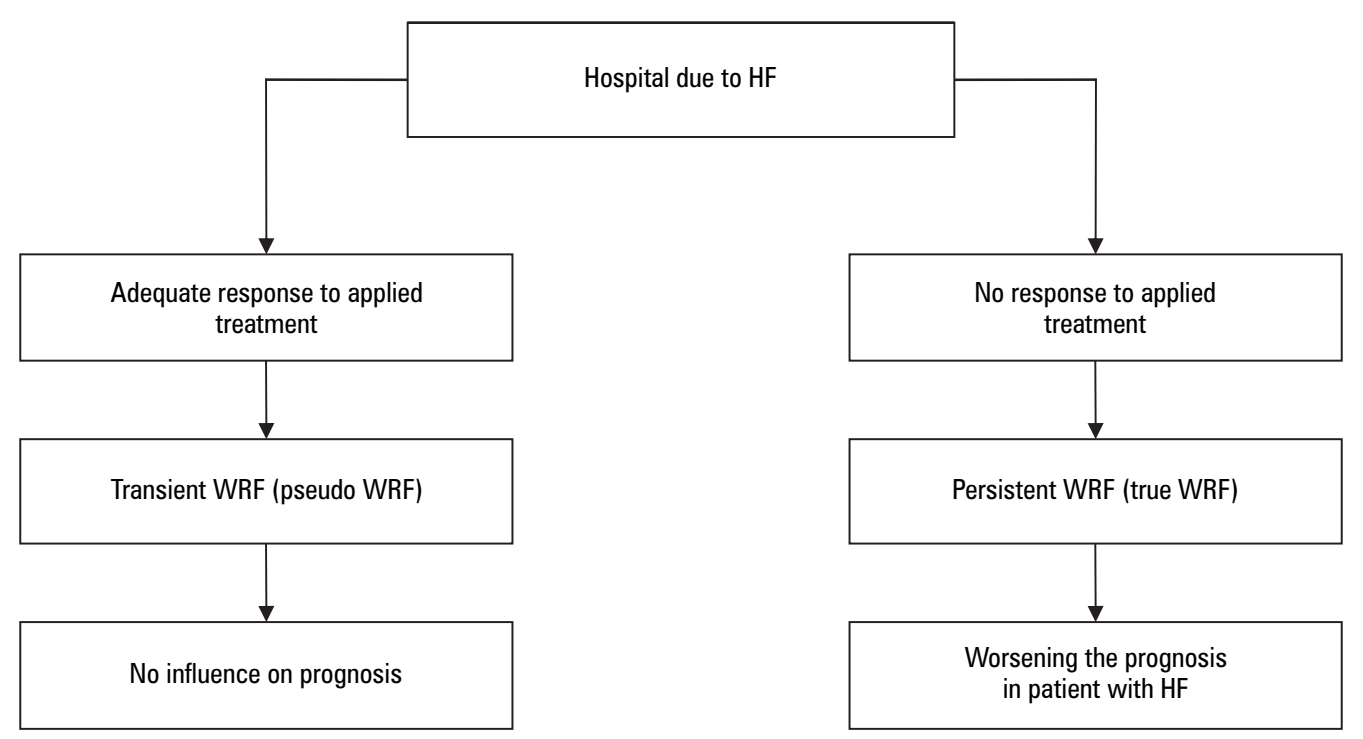

Figure 1. Forms of worsening renal function (WRF) in patients with heart failure (HF) [36]

with heart failure, $32 \%$ of patients had CKD associated with a 2-fold increased risk of mortality due to any cause. In an additional analysis of approximately 50000 patients with HF, WRF occurred in $23 \%$ of hospitalized patients and was associated with a 1.5 -fold higher risk of death due to any cause. CKD, diabetes, hypertension and the use of diuretics were proved to be significant risk factors for WRF in these patients [37]. In the literature, there are many definitions of WRF based on various absolute and relative values of serum creatinine increase [36]. The prognostic significance is not only the deterioration of kidney function in these patients but above all - its duration. In some hospitalized patients, WRF can only be of a temporary nature resulting from intensive therapy and does not necessarily indicate a worse prognosis for these patients [36].

In order to determine whether the WRF is temporary or permanent, Sheerin et al. [38] recommend that WRF is evaluated in patients with acute heart failure during hospitalization as well as for 3 months following discharge from hospital. WRF definition should differentiate the temporary decrease in glomerular filtration resulting from, among others, used pharmacotherapy and adequate response to treatment accompanied by clinical improvement [39]. In contrast to the chronically persistent decline in glomerular filtration as a result of, among others, too intensive treatment with a loop diuretic leading to haemodynamic and neurohormonal disorders and, as a consequence, to the development of cardiorenal syndrome (Fig. 1).

Deterioration of renal function occurs relatively often in patients with HF, when starting therapy with RAAS inhibitors and during further increase in their doses [34]. However, this should not dispose physicians to stop therapy with these drugs, as they provide long-term clinical benefits [40]. In a study conducted by Testani et al. [41] in the group of patients with left ventricular dysfunction, early WRF, in the subgroup where ACE-I enalapril was included in the treatment, no adverse prognostic significance was reported in contrast to the subgroup of patients with early WRF receiving placebo. In the placebo group, early WRF was associated with significantly increased mortality. A meta-analysis conducted by Clark et al. [40] covering over 20 thousand patients with left ventricular systolic dysfunction showed that in the group of patients in whom WRF was observed after initiating treatment with RAAS inhibitors, a significantly higher reduction in total mortality was found compared to the group of patients without WRF. Consequently, the WRF is not a prognostically homogeneous syndrome. Therefore, mechanism underlying WRF in patients with HF has a major prognostic significance.

Figures 2 and 3 present a practical approach to the treatment of patients with chronic heart failure and associated renal impairment, including ACE-Is or ARBs in therapy. 


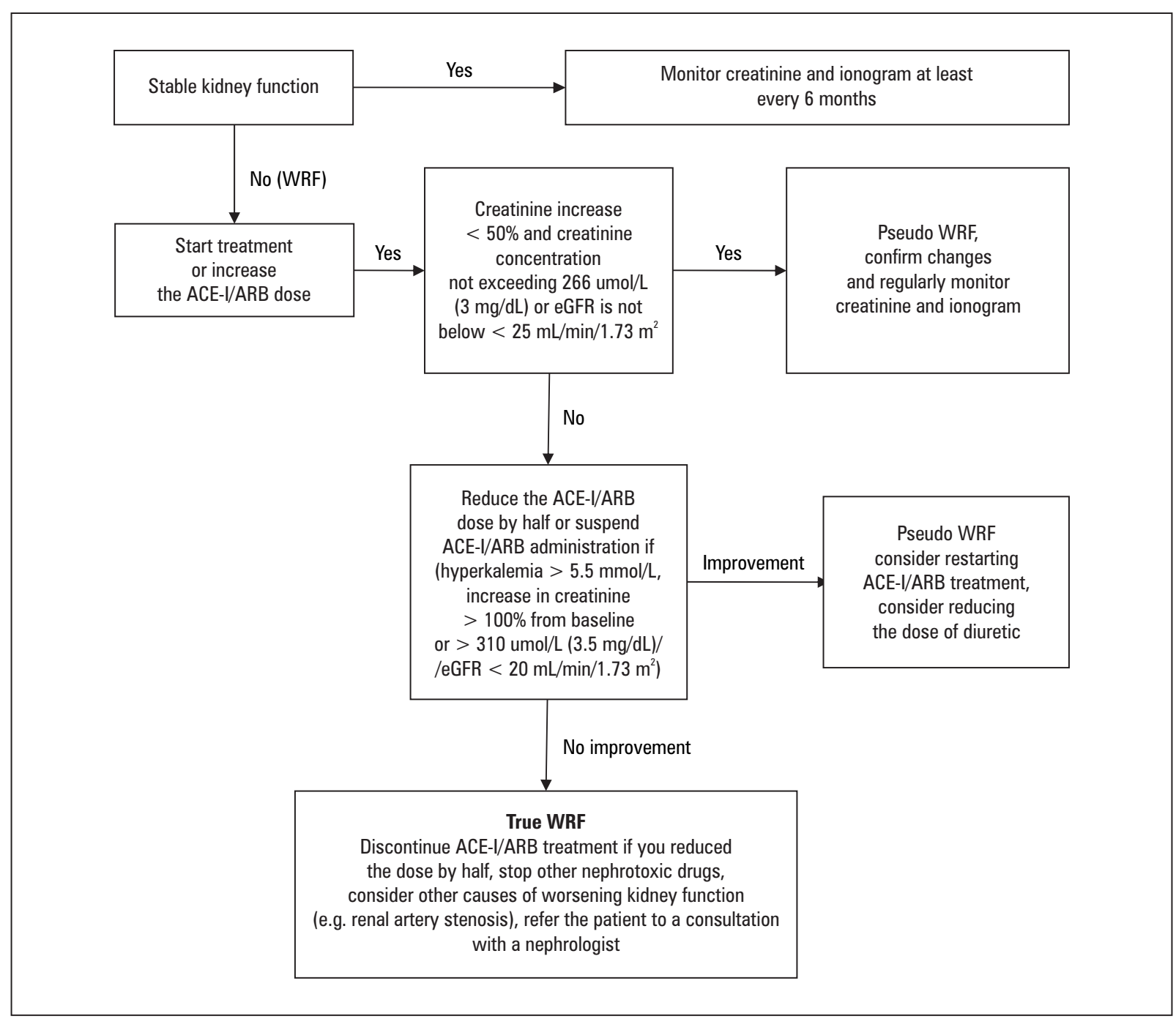

Figure 2. Practical approach to the treatment of patients with chronic heart failure (CHF) accompanied by impaired renal function - inclusion/intensification of ACE-Is or ARBs treatment. WRF — worsening renal function [37]

\section{Role of dual RAAS blockade in hypertension treatment}

Drugs from the ACE-Is or ARBs group constitute an essential component of first-line antihypertensive therapy in the basic treatment algorithm [42]. Both classes of drugs are the most commonly used antihypertensive drugs. The use of ARBs is associated with a significantly lower percentage of patients discontinuing treatment due to side effects compared to other classes of drugs and with a similar frequency of drug discontinuation compared to placebo [43]. According to the ESH/ESC guidelines for the management of hypertension (2018) and the guidelines of the Polish Society of Hypertension (2019), the simultaneous use of ACE-Is and ARBs is not recommended.

\section{RAAS blockade treatment in the era of coronavirus disease 2019 (Covid-19)}

It is worth to mention that human severe acute respiratory syndrome coronavirus 2 (SARS-Cov-2) binds to target cells through angiotensin-converting enzyme 2 (ACE-2) which expression can be found in respiratory epithelium and in epithelial cells of intestine, kidney, and blood vessels. The expression of ACE- 2 is substantially increased in patients with diabetes mellitus, hypertension, cardiovascular diseases who are treated with ACE-Is or ARBs. Angiotensin-converting enzyme 2 expression can also be increased by ibuprofen and thiazolidinediones. It was hypothesized that diabetes and hypertension treatment with ACE-2 stimulating drugs can be responsible for bad prognosis in COVID-19. There were even some suggestions that 


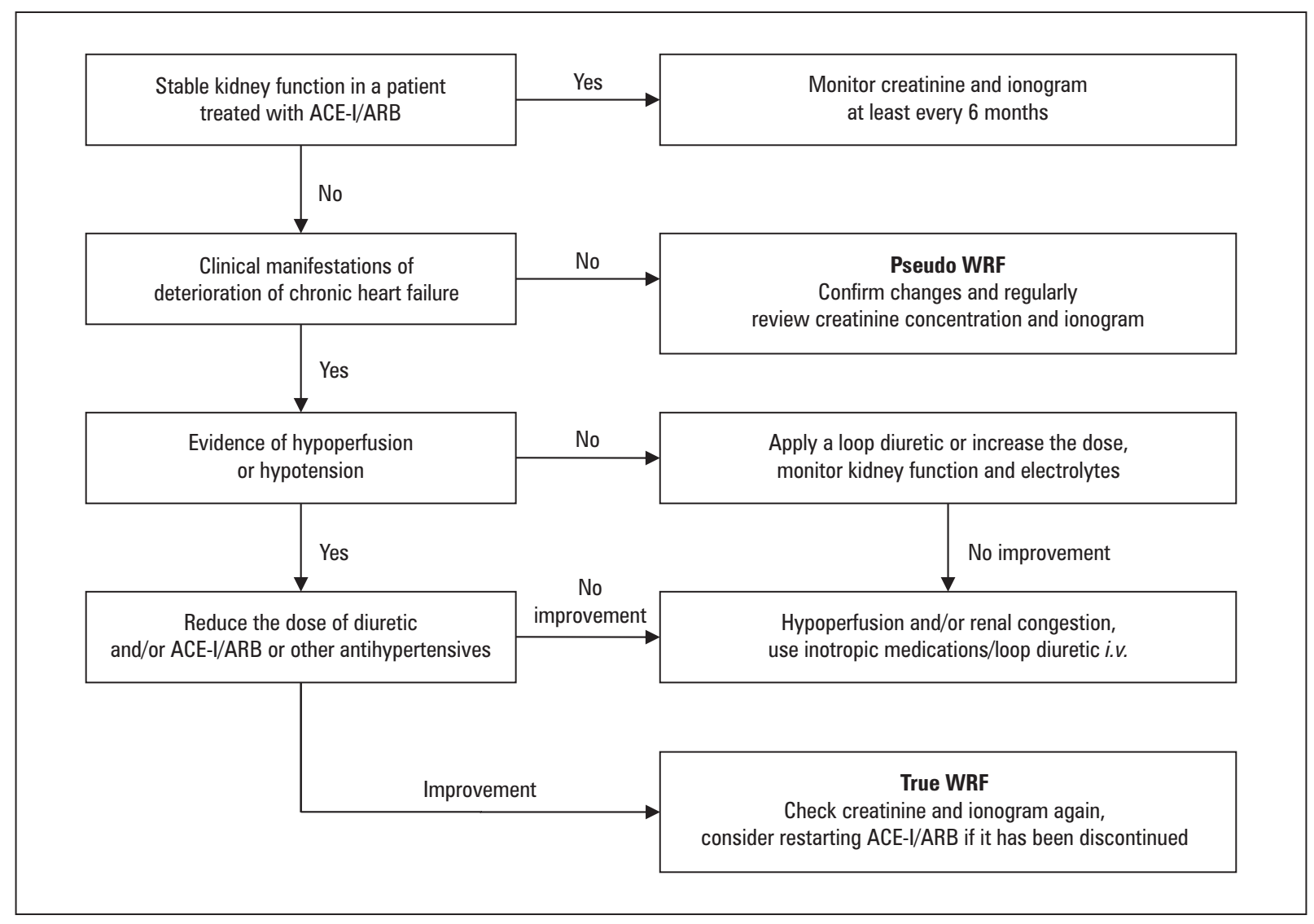

Figure 3. Practical approach to the treatment of patients with chronic heart failure accompanied by impaired renal function — indications for withdrawal or dose reduction of ACE-Is or ARBs. WRF — worsening renal function [37]

calcium channel blockers which do not act on RAAS should be first-line agents in hypertensive patients during COVID-19 pandemic [44]. On the other hand, ACE- 2 can be protective agent and in animal model of severe lung injury, ACE-Is or ARBs treatment inhibits the extent of lung injury. Reynolds et al. did not find any association between ACE-Is or ARBs treatment and severe clinical course of Covid-19 [45]. The same conclusion was made by Mehra et al. who did not confirm previous concerns regarding a potential harmful association of ACE inhibitors or ARBs with increased in-hospital mortality in COVID-19 [46]. The ACE-Is and $A R B s$ usage is more frequent among patients with COVID-19 because they are mainly older patients with higher prevalence of cardiovascular disease. There is a lack of evidence that ACE-Is or ARBs affect the risk of COVID-19 [47, 48]. An official ESH statement on COVID-19 from $15^{\text {th }}$ April 2020 concerning ACE-Is or ARBs treatment in COVID-19 pandemic strongly suggests no need for discontinuation of such treatment in stable patients with COVID-19 infection or at risk for COVID-19 infection. The treatment with ACE-Is and ARBs should be executed accord- ing to the recommendations in the $2018 \mathrm{ESC} / \mathrm{ESH}$ guidelines [49].

\section{References}

1. Khan M, Fonarow G, Khan H, et al. Renin-angiotensin blockade in heart failure with preserved ejection fraction: a systematic review and meta-analysis. ESC Heart Fail. 2017; 4(4): 402-408, doi: 10.1002/ ehf2.12204, indexed in Pubmed: 28869332.

2. Chalmers J, MacMahon S. Perindopril pROtection aGainst REcurrent Stroke Study (PROGRESS): interpretation and implementation. J Hypertens Suppl. 2003; 21: S9-S14, doi: 10.1097/00004872200306005-00003, indexed in Pubmed: 12953850.

3. Martínez-Milla J, García M, Urquía M, et al. Blockade of Renin-Angiotensin-Aldosterone System in Elderly Patients with Heart Failure and Chronic Kidney Disease: Results of a SingleCenter, Observational Cohort Study. Drugs Aging. 2019; 36(12): 1123-1131, doi: 10.1007/s40266-019-00709-1, indexed in Pubmed: 31493202.

4. Gayat E, Hollinger A, Cariou A, et al. Impact of angiotensin-converting enzyme inhibitors or receptor blockers on post-ICU discharge outcome in patients with acute kidney injury. Intensive Care Med. 2018; 44(5): 598-605, doi: 10.1007/s00134-018-5160-6, indexed in Pubmed: 29766216.

5. Jones M, Tomson C. Acute kidney injury and 'nephrotoxins': mind your language. Clin Med (London). 2018; 18(5): 384-386, doi: $10.7861 /$ clinmedicine.18-5-384, indexed in Pubmed: 30287431. 
6. Tomson C, Tomlinson L. Stopping RAS Inhibitors to Minimize AKI. Clin J Am Soc Nephrol. 2019; 14(4): 617-619, doi: 10.2215/ cjn.14021118, indexed in Pubmed: 30814113.

7. Momoniat T, Ilyas D, Bhandari S. ACE inhibitors and ARBs: Managing potassium and renal function. Clev Clin J Med. 2019; 86(9): 601-607, doi: 10.3949/ccjm.86a.18024, indexed in Pubmed: 31498767.

8. Hiremath S, Kayibanda J, Chow B, et al. Drug discontinuation before contrast procedures and the effect on acute kidney injury and other clinical outcomes: a systematic review protocol. Syst Rev. 2018; 7(1), doi: 10.1186/s13643-018-0701-1, indexed in Pubmed: 29467030.

9. Coca SG, Garg AX, Swaminathan M, et al. Preoperative angiotensinconverting enzyme inhibitors and angiotensin receptor blocker use and acute kidney injury in patients undergoing cardiac surgery. Nephrol Dial Transplant. 2013; 28(11): 2787-2799, doi: 10.1093/ ndt/gft405, indexed in Pubmed: 24081864.

10. Whiting P, Morden A, Tomlinson L, et al. What are the risks and benefits of temporarily discontinuing medications to prevent acute kidney injury? A systematic review and meta-analysis. BMJ Open. 2017; 7(4): e012674, doi: 10.1136/bmjopen-2016-012674, indexed in Pubmed: 28389482.

11. Hollmann C, Fernandes N, Biccard B. A Systematic Review of Outcomes Associated With Withholding or Continuing Angiotensin-Converting Enzyme Inhibitors and Angiotensin Receptor Blockers Before Noncardiac Surgery. Anest Analg. 2018; 127(3): 678-687, doi: 10.1213/ane.0000000000002837, indexed in Pubmed: 29381513

12. Bomback AS, Toto R. Dual Blockade of the Renin-AngiotensinAldosterone System: Beyond the ACE Inhibitor and AngiotensinII Receptor Blocker Combination. Am J Hypertens. 2009; 22(10): 1032-1040, doi: 10.1038/ajh.2009.138, indexed in Pubmed: 19661925.

13. Ruggenenti P, Perna A, Remuzzi G, et al. ACE inhibitors to prevent end-stage renal disease: when to start and why possibly never to stop: a post hoc analysis of the REIN trial results. Ramipril Efficacy in Nephropathy. J Am Soc Nephrol. 2001; 12(12): 2832-2837, indexed in Pubmed: 11729254.

14. Jr JW. Effect of Blood Pressure Lowering and Antihypertensive Drug Class on Progression of Hypertensive Kidney DiseaseResults From the AASK Trial. JAMA. 2002; 288(19): 2421-2431, doi: 10.1001/ jama.288.19.2421, indexed in Pubmed: 12435255.

15. Ruggenenti P, Fassi A, Ilieva A, et al. Preventing Microalbuminuria in Type 2 Diabetes. N Engl J Med. 2004; 351(19): 1941-1951, doi: 10.1056/nejmoa042167, indexed in Pubmed: 15516697.

16. Lewis E, Hunsicker L, Clarke W, et al. Renoprotective Effect of the Angiotensin-Receptor Antagonist Irbesartan in Patients with Nephropathy Due to Type 2 Diabetes. N Engl J Med. 2001; 345(12): 851-860, doi: $10.1056 /$ nejmoa011303, indexed in Pubmed: 11565517.

17. Brenner B, Cooper M, Zeeuw Dde, et al. Effects of Losartan on Renal and Cardiovascular Outcomes in Patients with Type 2 Diabetes and Nephropathy. N Engl J Med. 2001; 345(12): 861-869, doi: 10.1056/nejmoa011161, indexed in Pubmed: 11565518.

18. Barnett A, Bain S, Bouter P, et al. Angiotensin-Receptor Blockade versus Converting-Enzyme Inhibition in Type 2 Diabetes and Nephropathy. N Engl J Med. 2004; 351(19): 1952-1961, doi: 10.1056/ nejmoa042274, indexed in Pubmed: 15516696.

19. Struthers A. The clinical implications of aldosterone escape in congestive heart failure. Eur J Heart Fail. 2004; 6(5): 539-545, doi: 10.1016/j. ejheart.2004.04.013, indexed in Pubmed: 15301999.

20. Bomback A, Klemmer P. The incidence and implications of aldosterone breakthrough. Nat Clin Pract Nephrol. 2007; 3(9): 486-492, doi: 10.1038/ncpneph0575, indexed in Pubmed: 17717561.

21. Sato A, Hayashi K, Naruse M, et al. Effectiveness of Aldosterone Blockade in Patients With Diabetic Nephropathy. Hypertension. 2003; 41(1): 64-68, doi: 10.1161/01.hyp.0000044937.95080. e9, indexed in Pubmed: 12511531

22. Rossing K, Schjoedt KJ, Jensen BR, et al. Enhanced Renoprotective Effects of Ultrahigh Doses of Irbesartan in Patients With Type 2 Diabetes and Microalbuminuria. Kidney Int. 2005; 68(3):
1190-1198, doi: 10.1111/j.1523-1755.2005.00511.x, indexed in Pubmed: 16105050.

23. Schmieder R, Klingbeil A, Fleischmann E, et al. Additional Antiproteinuric Effect of Ultrahigh Dose Candesartan: A DoubleBlind, Randomized, Prospective Study. J Am Soc Nephrol. 2005; 16(10): 3038-3045, doi: 10.1681/asn.2005020138, indexed in Pubmed: 1612082.

24. Adamczak M. Reversal of Glomerulosclerosis after High-Dose Enalapril Treatment in Subtotally Nephrectomized Rats. J Am Soc Nephrol. 2003; 14(11): 2833-2842, doi: 10.1097/01. asn.0000095248.91994.d3, indexed in Pubmed: 14569093.

25. Donderski R, Manitius J. The role of aldosterone in kidney diseases and hypertension. Is it worth using mineralocorticoid receptor antagonists in clinical practice? Arterial Hypertension. 2019; 23(1): 1-7, doi: 10.5603/ah.a2018.0016.

26. Ruggenenti P. Dual renin-angiotensin system blockade for nephroprotection. Néphrol Thér. 2017; 13(Suppl 1): S43-S45, doi: 10.1016/j. nephro.2017.02.006, indexed in Pubmed: 28577742.

27. Čertíková Chábová VČ, Červenka L. The Dilemma of Dual ReninAngiotensin System Blockade in Chronic Kidney Disease: Why Beneficial in Animal Experiments But Not in the Clinic? Physiol Res. 2017; 66(2): 181-192, doi: 10.33549/physiolres.933607, indexed in Pubmed: 28471687.

28. Mogensen CE, Neldam S, Tikkanen I, et al. Randomised controlled trial of dual blockade of reninangiotensin system in patients with hypertension, microalbuminuria, and noninsulin dependent diabetes: the candesartan and lisinopril microalbuminuria (CALM) study. BMJ. 2000; 321(7274): 1440-1444, doi: 10.1136/ bmj.321.7274.1440, indexed in Pubmed: 11110735.

29. Mann J, Schmieder R, McQueen M, et al. Renal outcomes with telmisartan, ramipril, or both, in people at high vascular risk (the ONTARGET study): a multicentre, randomised, double-blind, controlled trial. Lancet. 2008; 372(9638): 547-553, doi: 10.1016/ s0140-6736(08)61236-2, indexed in Pubmed: 18707986.

30. Parving HH, Brenner B, McMurray J, et al. Cardiorenal End Points in a Trial of Aliskiren for Type 2 Diabetes. N Engl J Med. 2012; 367(23): 2204-2213, doi: 10.1056/nejmoa1208799, indexed in Pubmed: 23121378 .

31. Fried L, Emanuele N, Zhang J, et al. Combined Angiotensin Inhibition for the Treatment of Diabetic Nephropathy. N Engl J Med. 2013; 369(20): 1892-1903, doi: 10.1056/nejmoa1303154, indexed in Pubmed: 24206457.

32. Rutkowski B, Tylicki L. Nephroprotective Action of Renin-Angiotensin-Aldosterone System Blockade in Chronic Kidney Disease Patients: The Landscape After ALTITUDE and VA NEPHROND Trails. J Ren Nutr. 2015; 25(2): 194-200, doi: 10.1053/j. jrn.2014.10.026, indexed in Pubmed: 25576239.

33. Palevsky P, Zhang J, Seliger S, et al. Incidence, Severity, and Outcomes of AKI Associated with Dual Renin-Angiotensin System Blockade. Clin J Am Soc Nephrol. 2016; 11(11): 1944-1953, doi: 10.2215/cjn.03470316, indexed in Pubmed: 27679519.

34. Ponikowski P, Voors A, Anker S, et al. 2016 ESC Guidelines for the diagnosis and treatment of acute and chronic heart failure. Eur J Heart Fail. 2016; 18(8): 891-975, doi: 10.1002/ejhf.592, indexed in Pubmed: 27207191

35. Effects of Enalapril on Mortality in Severe Congestive Heart Failure. N Engl J Med. 1987; 316(23): 1429-1435, doi: 10.1056/ nejm198706043162301, indexed in Pubmed: 2883575.

36. Filippatos G, Farmakis D, Parissis J. Renal dysfunction and heart failure: things are seldom what they seem. Eur Heart J. 2013; 35(7): 416-418, doi: 10.1093/eurheartj/eht515, indexed in Pubmed: 24334714

37. Damman K, Valente M, Voors AA, et al. Renal impairment, worsening renal function, and outcome in patients with heart failure: an updated meta-analysis. Eur Heart J. 2013; 35(7): 455-469, doi: 10.1093/eurheartj/eht386, indexed in Pubmed: 24164864.

38. Sheerin N, Newton P, Macdonald P, et al. Worsening renal function in heart failure: The need for a consensus definition. Int J Cardiol. 2014; 174(3): 484-491, doi: 10.1016/j.ijcard.2014.04.162, indexed in Pubmed: 24801076. 
39. Damman K, Testani J. The kidney in heart failure: an update. Eur Heart J. 2015; 36(23): 1437-1444, doi: 10.1093/eurheartj/ehv010, indexed in Pubmed: 25838436.

40. Clark H, Krum H, Hopper I. Worsening renal function during reninangiotensin-aldosterone system inhibitor initiation and long-term outcomes in patients with left ventricular systolic dysfunction. Eur J Heart Fail. 2013; 16(1): 41-48, doi: 10.1002/ejhf.13, indexed in Pubmed: 24453097.

41. Testani J, Kimmel S, Dries D, et al. Prognostic Importance of Early Worsening Renal Function After Initiation of Angiotensin-Converting Enzyme Inhibitor Therapy in Patients With Cardiac Dysfunction. Circ Heart Fail. 2011; 4(6): 685-691, doi: 10.1161/circheartfailure.111.963256, indexed in Pubmed: 21903907.

42. Tykarski A, Filipiak KJ, Januszewicz A, et al. Zasady postępowania w nadciśnieniu tętniczym - 2019 rok. Wytyczne Polskiego Towarzystwa Nadciśnienia Tętniczego. Nadciśn Tętn Prakt. 2019; 5(1): 1-86.

43. Grupa Robocza Europejskiego Towarzystwa Kardiologicznego (ESC) i Europejskiego Towarzystwa Nadciśnienia Tętniczego (ESH) do spraw postępowania w nadciśnieniu tętniczym. Wytyczne ESC/ESH dotyczące postępowania w nadciśnieniu tętniczym. Kardiol Pol. 2019; 77(2): 71-159, doi: 10.5603/KP.2019.0018.
44. Fang L, Karakiulakis G, Roth M. Are patients with hypertension and diabetes mellitus at increased risk for COVID-19 infection? Lancet Respir Med. 2020; 8(4): e21, doi: 10.1016/s2213-2600(20)301168, indexed in Pubmed: 32171062.

45. Reynolds HR, Adhikari S, Pulgarin C, et al. Renin-Angiotensin-Aldosterone System Inhibitors and Risk of Covid-19. N Engl J Med. 2020; NEJMoa2008975, doi: 10.1056/NEJMoa2008975, indexed in Pubmed: 32356628.

46. Mehra M, Desai S, Kuy S, et al. Cardiovascular Disease, Drug Therapy, and Mortality in Covid-19. N Engl J Med. 2020; NEJMoa2007621, doi: 10.1056/nejmoa2007621, indexed in Pubmed: 32356626.

47. Mancia G, Rea F, Ludergnani M, et al. Renin-Angiotensin-Aldosterone System Blockers and the Risk of Covid-19. N Engl J Med. 2020; NEJMoa2006923, doi: 10.1056/nejmoa2006923, indexed in Pubmed: 32356627.

48. Thomas G. Renin-angiotensin system inhibitors in COVID-19. Cleve Clin J Med. 2020, doi: 10.3949/ccjm.87a.ccc009, indexed in Pubmed: 32409434.

49. Statement of the European Society of Hypertension (ESH) on hypertension, Renin-Angiotensin System (RAS) blockers and COVID-19. https://www.eshonline.org/spotlights/esh-statementcovid-19/ (April 15th 2020) 